\title{
Arrhythmias in Rat Hearts Exposed to Pulsed Ultrasound After Intravenous Injection of a Contrast Agent
}

Abbreviations

FDA, Food and Drug Administration; HBFP, hematoxylin-basic fuchsin-picric acid; MI, mechanical index

Received May 7, 2002, from the Bioengineering Program, University of Illinois at Urbana-Champaign (J.F.Z., L.A.F., W.D.O.), Bioacoustics Research Laboratory, Department of Electrical and Computer Engineering, University of Illinois (S.A.H., L.A.F., W.D.O.), Urbana, Illinois. Revision requested June 13, 2002. Revised manuscript accepted for publication July 31, 2002.

We thank James P. Blue, Kandice $S$. Norrell, Rita J. Miller, Eric J. Wort, and Bill Zierfuss from the Bioacoustics Research Laboratory and the staff of the Histopathology Laboratory, College of Veterinary Medicine, University of Illinois, for technical contributions. We also thank Prof Douglas $G$. Simpson for consultation on the statistical analysis. This study was supported in part by National Institutes of Health grant HL58218 (W.D.O. and J.F.Z.).

Address correspondence and reprint requests to James F. Zachary, DVM, PhD, University of Illinois, 2001 S Lincoln Ave, Urbana, IL 61802.
James F. Zachary, DVM, PhD, Sarah A. Hartleben, BS, Leon A. Frizzell, PhD, William D. O'Brien, Jr, PhD

Objective. To develop an animal model suitable for characterizing electrocardiographic arrhythmias in hearts exposed to ultrasound after injection of a microbubble contrast agent. Methods. Conduction complex and heart lesion data were recorded from 20 rats that received intravenous injections of $0.25 \mathrm{~mL}$ of a contrast agent and were exposed to pulsed ultrasound (frequency, 3.1 MHz; pulse duration, 1.3 microseconds; pulse repetition frequency, $1700 \mathrm{~Hz}$; and in situ peak rarefactional pressure, $15.9 \mathrm{MPa}$ ). The volume of the contrast agent based on body weight and the mechanical index (ultrasonic pressure) exceeded those used in echocardiography by 14 to 345 and 3 to 29 times, respectively. Results. Premature atrial complexes, premature ventricular complexes, or polymorphic ventricular tachycardia occurred in 10 rats. When ultrasound exposure was halted, arrhythmias ceased but reoccurred in 4 of the 10 rats when exposure resumed. Myocardial degeneration identified by histochemical staining (hematoxylin-basic fuchsinpicric acid) was observed in 16 rats; however, only 10 rats had arrhythmias. There was no significant difference in the amount of histochemical staining in hearts from rats with arrhythmias when compared with rats without arrhythmias. Conclusions. An animal model suitable for characterizing electrocardiographic arrhythmias in rat hearts exposed to ultrasound after injection of a microbubble contrast agent was developed. Because arrhythmias were induced principally when the contrast agent interacted with ultrasound during exposure, the presence of myocardial degeneration alone was not a sufficient explanation for ectopic electrical activity. Under these extreme exposure conditions, the data suggest that pulsed ultrasound through its biomechanical interactions with contrast agents has the potential to induce arrhythmias. Key words: arrhythmias; conduction abnormalities; contrast agent; pulsed ultrasound.

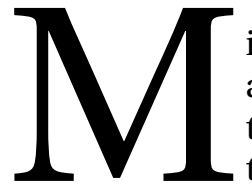
icrobubble contrast agents are used as adjuncts in echocardiographic examinations to increase the opportunity for early detection and diagnosis of heart disease. ${ }^{1-6}$ Intravenous contrast-enhanced echocardiography provides the following potential benefits: (1) improved image quality, especially in patients in whom sonograms are suboptimal because of obesity or lung disease; (2) enhanced visualization of ventricular opacification and endocardial border delineation; and (3) improved image acquisition 
and enhanced delineation of regional wall motion abnormalities at the peak level of exercise in stress echocardiography. ${ }^{2}$ Furthermore, assessment of myocardial perfusion and coronary artery disease is likely to become a useful diagnostic tool to measure global and regional myocardial structure, function, and perfusion. ${ }^{2}$

When administered intravenously, contrast agents are effective because they substantially enhance the distinction between tissues with similar acoustic impedances such as the ventricular myocardium and the blood within the ventricle. ${ }^{6}$ Contrast enhancement results from increased reflection of ultrasound from microbubbles circulating in blood vessels, vascular beds, and heart chambers when compared with adjacent tissue. ${ }^{7}$

Concerns have been raised about the interaction of ultrasound with microbubble contrast agents in studies documenting in vitro and in vivo bioeffects. ${ }^{8-20}$ These concerns have been addressed by the Bioeffects Committee of the American Institute of Ultrasound in Medicine. ${ }^{21,22}$ It was advised that contrast agent exposure conditions that minimize the potential for bioeffect occurrence should be used. However, the consensus statements did not provide guidelines as to what exposure conditions would minimize the potential for bioeffect occurrence. Subsequent to these consensus statements, premature ventricular contractions were reported in healthy adult humans during triggered secondharmonic imaging of a contrast agent for myocardial perfusion. ${ }^{23}$

The purpose of this study was to develop an animal model suitable for characterizing electrocardiographic arrhythmias in hearts exposed to ultrasound after intravenous injection of a microbubble contrast agent. If an appropriate animal model could reproduce ultrasoundinduced electrocardiographic arrhythmias with contrast agent concentrations and exposure conditions at levels much in excess of those used clinically, then future studies could be designed to assess threshold levels. If not, then there would be no need to pursue further studies. The purpose was fulfilled by (1) determining whether arrhythmias could be induced in rat hearts exposed to pulsed ultrasound after intravenous contrast agent injection, (2) determining the total number of arrhythmias per minute of treatment under a variety of treatment conditions, (3) determining the character of arrhythmias, and (4) correlating arrhythmias with myocardial degeneration as identified by histochemical staining of heart tissue.

\section{Materials and Methods}

\section{Experimental Design}

Ultrasound exposure conditions and the volume of the contrast agent administered based on body weight in this study greatly exceeded those used routinely in contrast agent-enhanced echocardiography. The volume of the contrast agent based on body weight and the mechanical index (MI; ultrasonic pressure) exceeded those used in echocardiography by 14 to 345 and 3 to 29 times, respectively.

\section{Animals}

The experimental protocol was approved by the Laboratory Animal Care Advisory Committee, University of Illinois at Urbana-Champaign, and satisfied all campus and National Institutes of Health rules for the humane use of laboratory animals.

Rats were chosen as the experimental animals on the basis of expertise acquired from our previous studies with lungs that examined ultrasound-induced bioeffects. ${ }^{24-27}$ The hearts of 10- to 11-week-old $269 \pm 12$-g (mean \pm SD) female Sprague Dawley rats (Harlan, Indianapolis, IN) were exposed in vivo through the sternum to pulsed ultrasound after intravenous injection of a contrast agent. These 20 rats were evaluated by electrocardiography for arrhythmias and by histopathologic examination for myocardial injury. Fifteen additional 10- to 11-week-old $251 \pm 15$-g female Sprague Dawley rats were used as control rats for the histopathologic assessment of myocardial injury.

Rats were weighed and anesthetized with ketamine hydrochloride $(87.0 \mathrm{mg} / \mathrm{kg})$ and xylazine $(13.0 \mathrm{mg} / \mathrm{kg})$ administered intraperitoneally. Anesthetized rats were placed in dorsal recumbency. Respiratory rates and peripheral vascular perfusion (ear skin color and mucous membrane color) were monitored visually. Experiments were conducted in a room maintained at $22^{\circ} \mathrm{C}$. To minimize body heat loss to an adjacent tabletop, rats were placed on a thick insulating paper barrier and in contact with the thin membrane of the $30^{\circ} \mathrm{C}$ standoff tank (Fig. 1C). The skin over the sternum was exposed by removing the hair 


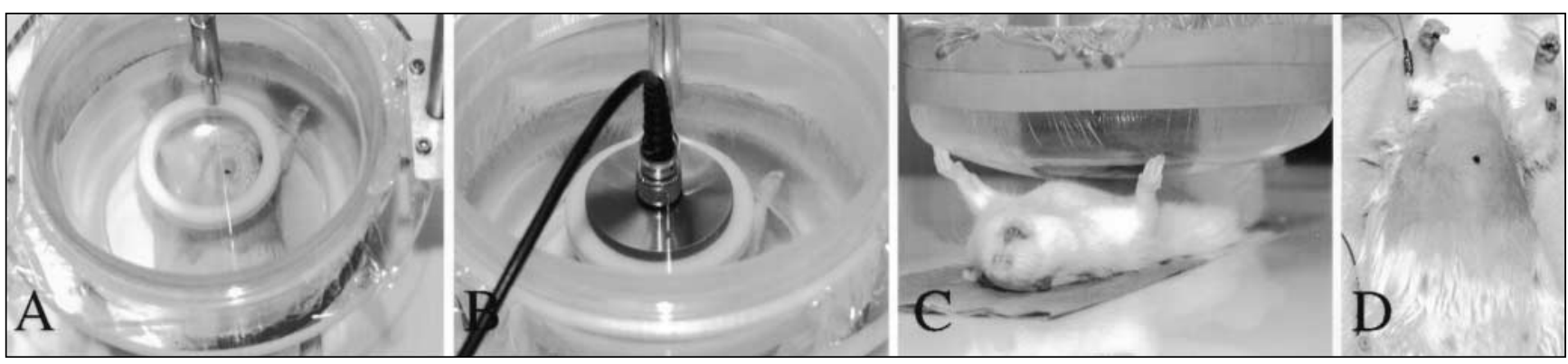

Figure 1. Rat preparation and transducer alignment. A, After depilation, rats were placed in dorsal recumbency. A black dot was placed on the skin over the sternum to mark the site for alignment of the ultrasonic transducer. B, The focused 51-mm-diameter lithium niobate ultrasonic transducer was aligned perpendicular to the black dot. C, A standoff vessel containing degassed water $\left(30^{\circ} \mathrm{C}\right)$ was placed in contact with the skin of the rat. The membrane of the standoff vessel made direct contact with the skin surface. A small quantity of mineral oil was used to ensure complete contact between the membrane and the skin. D, Electrocardiographic leads were attached to the limbs in a lead I configuration before the alignment procedure.

with an electric clipper, followed by a depilatory agent (Nair; ArmKel, LLC, Princeton, NJ) to maximize sound transmission. An intravenous 25-gauge catheter was inserted into the lateral tail vein and taped in place. A black dot was placed on the skin over the sternum at approximately $1.7 \mathrm{~cm}$ cranial to the xyphoid process to guide the positioning of the transducer's ultrasonic beam (Fig. 1, A and D). A standoff tank was positioned in contact with the skin with the use of mineral oil as a coupling agent (Fig. 1C). A circular transducer holder was visually centered above the black dot (Fig. 1A). The transducer was placed in the holder in the standoff tank, which contained highly degassed water at $30^{\circ} \mathrm{C}$ (Fig. 1B). The low-power pulse-echo capability of the exposure system (RAM5000; Ritec Inc, Warwick, RI), displayed on a digital oscilloscope (9354CTM; LeCroy, Chestnut Ridge, NY), was used to adjust the calibrated transducer's focal region center $6 \mathrm{~mm}$ posterior to the skin surface echo (the focal region length [the -6-dB pulse-echo depth of focus] was $5.9 \mathrm{~mm}$ ). Fine tuning of the transducer's position was then done until 3 distinct echoes were seen within the focal region (Fig. 2, A and B). The ultrasonic beam axis was approximately perpendicular to the heart at the position of the black dot, with the beam's focal region within the heart. The oscilloscope's echo signals were also used to visually determine whether the ultrasonic field interacted with the contrast agent within the circulatory system during exposure (Fig. 2C). For those rats in which contrast agents were injected, the echo signals were greatly increased.

\section{Electrocardiography}

Electrocardiographic electrodes were inserted into the skin to produce a lead I configuration (Fig. 1D). Electrocardiograms were recorded digitally with an SRA-400b sinus rhythm analyzer (Micro-Med Inc, Louisville, KY). Electrocardiograms were collected continuously in the following sequential order: (1) normal sinus rhythm, (2) ultrasound exposure alone, (3) contrast agent alone, (4) ultrasound and contrast agent, (5) contrast agent alone, (6) ultrasound and contrast agent, and (7) contrast agent alone. Electrocardiographic recordings were collected continuously from the onset of treatment (baseline normal sinus rhythm) until the recording session was terminated. Recording times of approximately 2 minutes' duration were attempted in each of the treatment conditions listed above; however, the complexity of the technical and recording procedures hindered attainment of precisely timed intervals. The number and character of arrhythmias were correlated with the treatment conditions for each recording interval described above. Results were expressed as the number of arrhythmias per minute of recording time (rate).

In rats that received intravenous injections of the contrast agent and were exposed to ultrasound, meaningful arrhythmias (more than 2 consecutive premature contractions) were observed when exposure was initiated. If no arrhythmias were observed during the first minute of experimental interval 4 (ultrasound and contrast agent), the duration of data collection was reduced or eliminated in subsequent experimental intervals. In rats that had development of meaningful arrhythmias in experimen- 


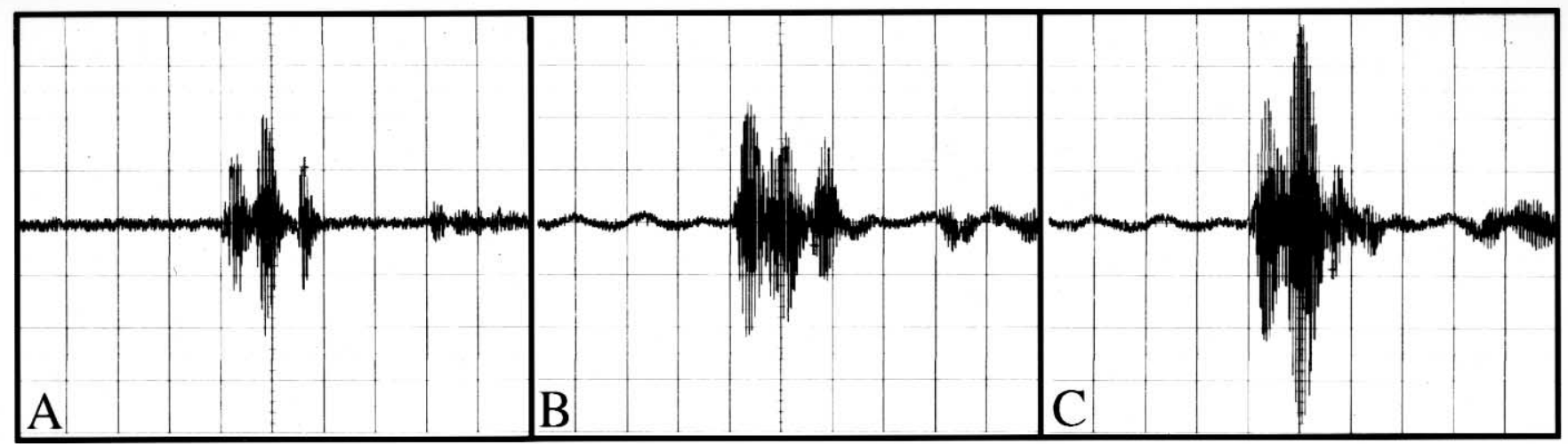

Figure 2. Example of the A-mode images seen during the alignment procedure (A), ultrasound exposure without the circulating contrast agent (B), and ultrasound exposure with the circulating contrast agent $(\mathbf{C})$. In $\mathbf{A}$, the chest wall (first 2 echoes), heart walls (third and fourth echoes), and heart chambers (between the third and fourth echoes) are shown; the first 3 echoes are located in the middle of the trace, and the fourth echo is located near the right side of the trace. Approximately 12 microseconds separated the third and fourth echoes. The time base was 5 rs/division (equivalent to $3.85 \mathrm{~mm} /$ division), and the amplitude was $100 \mathrm{mV} /$ division.

tal interval 4, electrocardiographic data were collected during a second exposure of the heart to ultrasound. It was known that Optison (FS069; Mallinckrodt Inc, St Louis, MO) would be cleared rapidly by the lungs after intravenous administration, and it was hypothesized that the occurrence of arrhythmias would decrease with a second exposure of the heart to ultrasound.

\section{Contrast Agent}

Optison, an injectable sonographic echogenic contrast agent that consists of hollow microspheres of heat-denatured human albumin filled with octafluoropropane, was used in this study. ${ }^{28}$ In humans, the recommended diagnostic dose is $0.5 \mathrm{~mL}$ with a total maximal dose of $8.7 \mathrm{~mL} .{ }^{29} \mathrm{In}$ this study, Optison was injected intravenously (0.25-mL total dose) as a bolus into the lateral tail vein, followed by $0.25 \mathrm{~mL}$ of $0.9 \% \mathrm{NaCl}$. This volume was used to ensure a reasonable probability of inducing arrhythmias.

The volume of Optison administered was based on product safety information provided in the package insert. ${ }^{29}$ In the product safety study, Optison was administered intravenously in rats during organogenesis at doses of 0.25 and $5.0 \mathrm{~mL}$ $\cdot \mathrm{kg}^{-1} \cdot \mathrm{d}^{-1}$ and was approximately 0.2 and 5.0 times the recommended maximal human dose based on body surface area. By extrapolation, a dose of $1.0 \mathrm{~mL} \cdot \mathrm{kg}^{-1} \cdot \mathrm{d}^{-1}$ would be approximately equal (1.0 $\times$ ) the recommended maximal human dose based on body surface area. The average weight of an adult Sprague Dawley rat used in this study was approximately $250 \mathrm{~g}(0.25 \mathrm{~kg})$; there- fore, $0.25 \mathrm{~mL}$ of Optison was administered intravenously to each rat.

\section{Pathologic Examination}

After treatment, each rat was killed by cervical dislocation while anesthetized. Hearts were examined macroscopically and then processed for histologic evaluation by routine methods. Sections were stained with hematoxylin-eosin for routine microscopic evaluation and hematoxylin-basic fuchsin-picric acid (HBFP) for inapparent myocardial degeneration. ${ }^{30-32}$ Each heart section was assigned a numerical HBFP staining score between 0 and 5 . A score of 0 was assigned when there was no HBFP staining. As the amount of HBFP staining increased, scores were assigned between 1 and 5 to reflect the incremental amounts of staining. Myocardial staining in both experimental and control rats was scored blindly.

\section{Exposimetry}

Ultrasound exposure conditions and transducer calibration procedures used in this study were described previously. ${ }^{24-27}$ In summary, a focused 51-mm-diameter lithium niobate ultrasonic transducer (ValpeyFisher Corporation, Hopkinton, MA) was used to expose each heart. The lowpower pulse-echo capability of the RAM5000 exposure system was used to align the transducer's axial position on the heart. The transducer was characterized and calibrated in degassed water $\left(22^{\circ} \mathrm{C}\right)$ according to established procedures. $^{27,33}$ The pulse-echo characterization procedure yielded a center frequency of $3.1 \mathrm{MHz}$, a 
fractional bandwidth of $15 \%$, a focal length of $56 \mathrm{~mm}, \mathrm{a}-6-\mathrm{dB}$ focal beam width of $610 \mu \mathrm{m}$, and a-6-dB depth of focus of $5.9 \mathrm{~mm}$. In comparison with the beam width of $610 \mu \mathrm{m}$, hearts measured approximately $1.5 \mathrm{~cm}$ in length and $1.0 \mathrm{~cm}$ in diameter. The calibration procedure used a calibrated polyvinylidene difluoride hydrophone (Y-34-6543; Marconi, Chelmsford, Essex, England) and yielded an in vitro peak rarefactional pressure of $20.2 \mathrm{MPa}$, an in vitro peak compressional pressure of $45.8 \mathrm{MPa}$, an MI of 5.8 , and a pulse duration of 1.3 microseconds ( 4 cycles). The in situ (at the heart) acoustic pressure was estimated by taking into account attenuation of 6-mm-thick interposed tissues $\left(1.1 \mathrm{~dB} \cdot \mathrm{cm}^{-1} \cdot \mathrm{MHz}^{-1}\right) .^{34}$

This estimate yielded at the center of the focal region (6 $\mathrm{mm}$ from the skin surface), an in situ peak rarefactional pressure of $15.9 \mathrm{MPa}$, and an in situ peak compressional pressure of $36.1 \mathrm{MPa}$. The exposure of each rat's heart was conducted at a pulse repetition frequency of $1700 \mathrm{~Hz}$. The magnitude of acoustic pressure selected was based on previous findings that produced ultrasound-induced lung hemorrhage. ${ }^{24-27}$ The exposure level was considerably greater than that allowed under current regulations. ${ }^{35}$ At the exposure conditions used, the equivalent MI was 5.8, whereas the regulatory limit is 1.9 for diagnostic ultrasonic equipment that falls under Food and Drug Administration (FDA) control, that is, 3 times greater than the FDA's regulatory MI limit. ${ }^{36}$

\section{Data Analysis}

The total number of abnormal complexes per treatment condition and per minute of treatment condition were summed for all exposed rats. Analysis of variance (single factor) was used to compare HBFP staining differences between control and treatment groups.

\section{Results}

\section{Electrocardiography}

Clinically meaningful arrhythmias developed in $10(50 \%)$ of 20 rats exposed to pulsed ultrasound after intravenous injection of the contrast agent. The number of arrhythmias for each treatment condition, the duration of each treatment condition, and the number of arrhythmias per minute for each treatment condition are summed and shown in Figure 3.
Five rats had a single arrhythmia during baseline data (normal sinus rhythm) collection. Ten rats had a total of 13 arrhythmias when exposed to ultrasound alone. These arrhythmias generally occurred as single events when ultrasound exposure was first initiated. Three of these 10 rats had 2 consecutive arrhythmias. Seven rats had a single arrhythmia that generally occurred during the time when the contrast agent was injected intravenously. Ten rats had a total of 131 arrhythmias when exposed to ultrasound while the contrast agent was circulating in the cardiovascular system. When ultrasound exposure was

Figure 3. Data summed for all 20 experimental rats. The number of arrhythmias for each treatment condition, the duration of each treatment condition, and the number of arrhythmias per minute for each treatment condition are shown. CA indicates contrast agent; SR, normal sinus rhythm (baseline); US, ultrasound exposure; and US \& CA, ultrasound exposure with the circulating contrast agent.
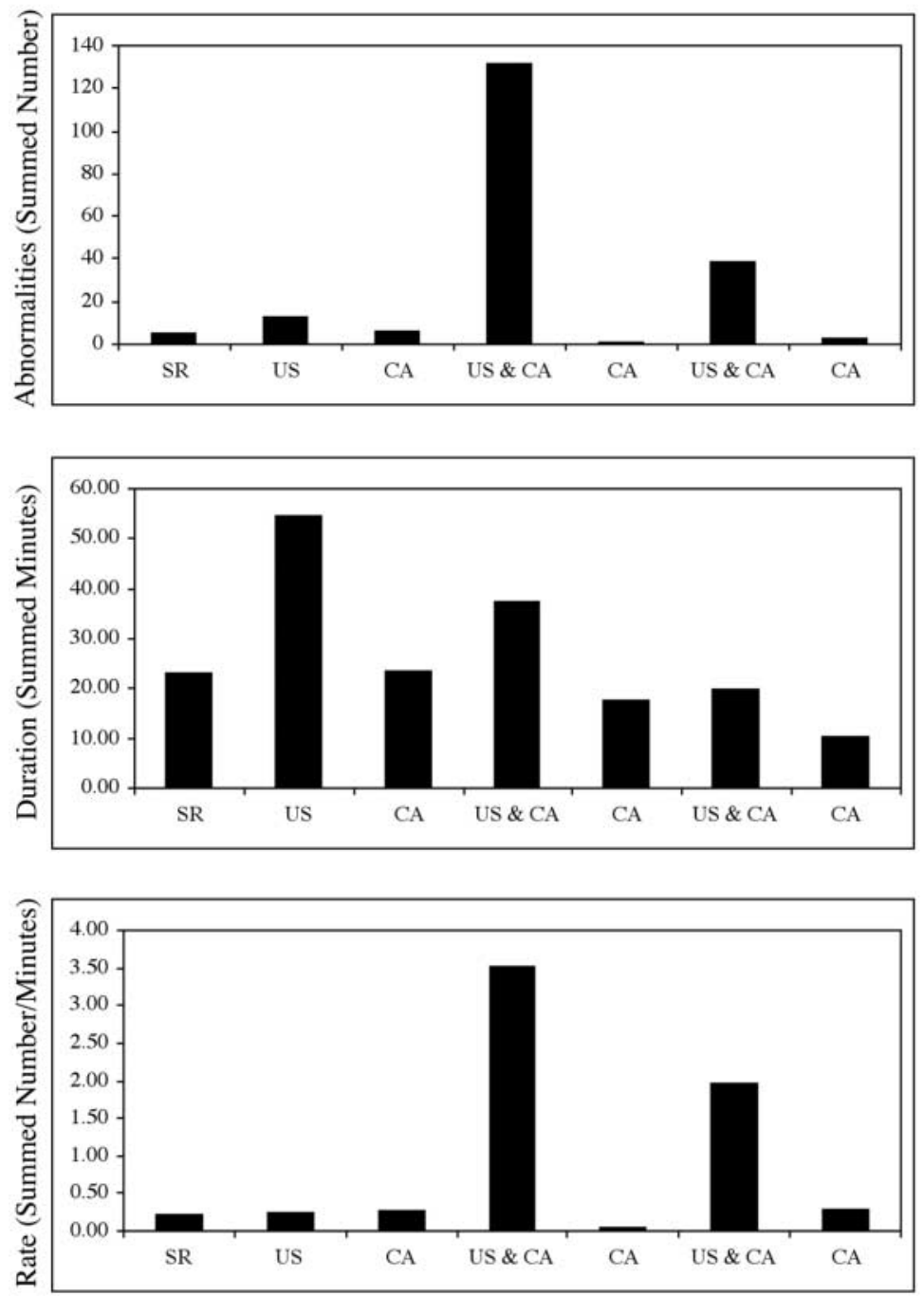
halted, arrhythmias ceased, and normal sinus rhythm returned in all but 2 rats. Sinus rhythm abnormalities developed in these 2 rats for the remainder of the recording time. Four rats that had arrhythmias during the first contrast agent-ultrasound exposure period had a total of 39 arrhythmias when ultrasound exposure resumed while the contrast agent was circulating in the cardiovascular system.

Arrhythmias included premature atrial contractions, premature ventricular contractions, and ventricular tachycardia (Fig. 4). In most rats, the configuration of arrhythmias was polymorphic and restricted solely to an origin in the atria or the ventricles. Continuous sinus rhythm abnormalities (atrial or ventricular bigeminy) developed in 2 rats after the initial onset of arrhythmias.

\section{Pathologic Findings}

Control rats $(n=5)$ not exposed to ultrasound or given an intravenous injection of the contrast agent had a mean \pm SD HBFP staining score of $0.6 \pm 0.55$. Control rats $(n=5)$ exposed to ultrasound had a mean staining score of $1.2 \pm 0.45$. Control rats $(n=5)$ not exposed to ultrasound but given an intravenous injection of the contrast agent had a mean staining score of $1.0 \pm 0.71$. Analysis of variance showed no significant differences among these 3 control groups $(P=.28)$. Thus, there were no differences in the degree of labeling (injury) of myocardial cells in rat hearts exposed to ultrasound or the contrast agent alone when compared with $0.9 \% \mathrm{NaCl}$ alone.
Analysis of variance showed no significant differences $(P=.85)$ in the degree of HBFP labeling (injury) of myocardial cells between rats with arrhythmias and rats without arrhythmias. These results suggested that the occurrence of arrhythmias was unrelated to the presence or absence of myocardial injury in the heart.

There was no statistically significant difference $(P=.12)$ in the degree of HBFP labeling (injury) of myocardial cells between control rats (including all 3 control groups, $\mathrm{n}=15$ ) and experimental rats $(n=20)$. These results suggest that (1) the interaction of ultrasound with the intravenous contrast agent did not induce differences in HBFP labeling (injury) of myocardial cells between these 2 groups; and (2) the occurrence of arrhythmias was again unrelated to the presence or absence of myocardial injury in the heart.

One rat (5\%) had a macroscopic lesion that extended transmurally through the myocardium of the right ventricular free wall to the endocardium on the ventricle (Fig. 5, A and B). There were no visible lesions in the interventricular septum or free wall of the left ventricle. Microscopic lesions were similar in character in control rats and in rats that had or did not have arrhythmias. In all rats with lesions, the lesions consisted of 1 or more foci of swollen, hyalinized necrotic rhabdomyocytes without an inflammatory response (acute coagulative necrosis) (Fig. 5, C and D).

Figure 4. Electrocardiographic data (lead I) from a rat exposed to pulsed ultrasound after intravenous injection of the contrast agent. Analysis of the electrocardiographic data showed normal sinus rhythm complexes (complexes to the left of the arrow). When the contrast agent was in the systemic circulation and ultrasound exposure was initiated (arrow), ventricular tachycardia was observed (complexes to the right of the arrow).

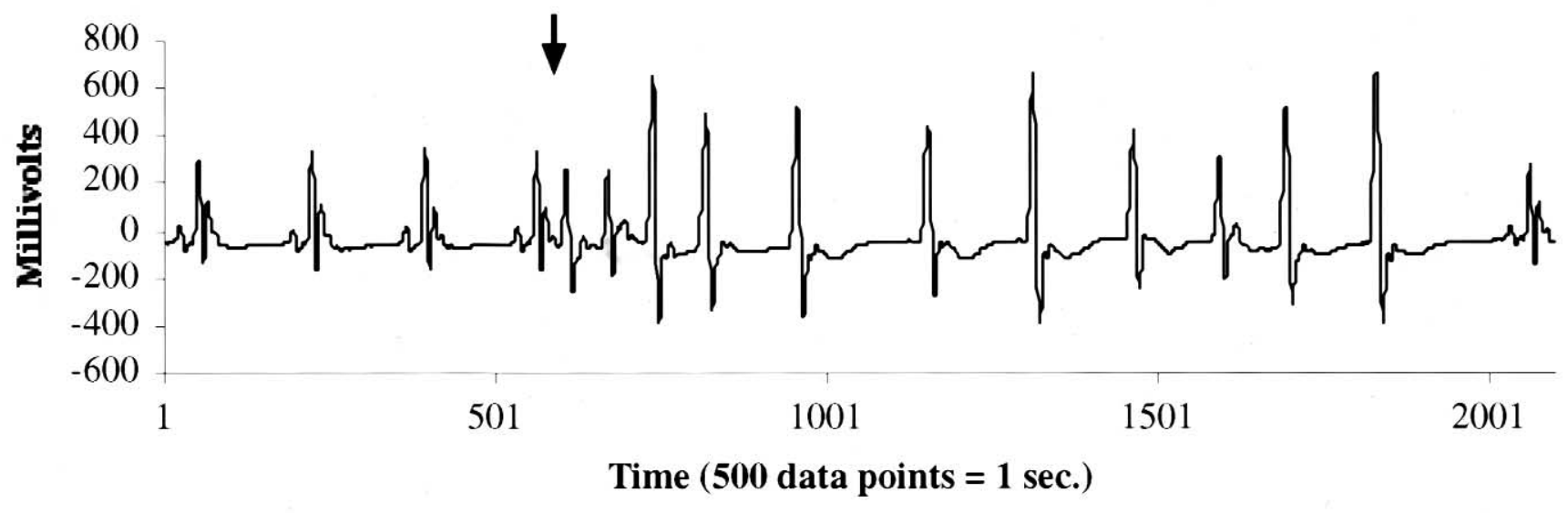




\section{Discussion}

In this study, we have shown that extremely high-powered pulsed ultrasound, through its biomechanical interactions with excessively large doses of a contrast agent, has the potential to induce arrhythmias. The volume of the contrast agent based on body weight and the MI (ultrasonic pressure) used in this study exceeded those recommended for use in echocardiography by 14 to 345 and 3 to 29 times, respectively. There were no clinically meaningful arrhythmias when the heart was in the sinus rhythm or when it was exposed to pulsed ultrasound alone or the contrast agent alone. The clinical relevance of these findings is as yet unknown.

The arrhythmias induced in our rats included atrial premature contractions, ventricular premature contractions, and ventricular tachycardia. Because echogenic contrast agents are used to improve diagnostic imaging in patients with heart disease that already have an increased risk of having fatal arrhythmias, the relationship between the use of these agents and the induction of cardiac arrhythmias must be thoroughly understood.

In our rats, arrhythmias were dissimilar in conformation and duration and thus were categorized as predominately polymorphic, suggesting that they likely originated from multiple foci within the right and left ventricles (ventricular premature contractions and ventricular tachycardia) or right and left atria (atrial premature contractions and abnormal sinus rhythms). The characteristics of the transmural lesion extending from the epicardium to the endocardium of the right ventricular free wall implied that the genesis of the lesion was related to the interaction of the contrast agent with the ultrasonic field as the bubbles passed through microvascular beds of the myocardium. In addition, the finding of microscopic myocardial lesions distributed at random within the ultrasonic field in the interventricular septum and right and left ventricular free walls suggests that the interaction of the contrast agent with the ultrasonic field also occurs within the same microvascular beds. The outcome of this interaction likely leads to injury of rhabdomyocytes in exposed areas; however, this lesion does not appear to contribute to the generation of arrhythmias.

The occurrence of 1 or 2 arrhythmias after exposure of hearts to pulsed ultrasound alone or the contrast agent alone as observed in our rats was not unexpected. Studies have shown that a single pulse of high-intensity pulsed ultrasound focused on the hearts of frogs, ${ }^{35,37,38}$ mice, ${ }^{39}$ and pigs $^{40}$ can cause single premature contractions.

Figure 5. Hematoxylin-basic fuchsin-picric acid staining in rats exposed to pulsed ultrasound. A, Ventral (sternum)-dorsal view of the heart after fixation in $10 \%$ buffered formalin. The heart had an area of acute myocardial injury aligned with the focal plane of the ultrasonic beam over the free wall of the right ventricle (arrow). This lesion was observed in only 1 (5.0\%) of 20 rats exposed to ultrasound. B, Midsagittal view of the transected heart after fixation in $10 \%$ buffered formalin. The heart had an area of acute myocardial injury aligned with the focal plane of the ultrasonic beam that extended from the epicardial surface through the free wall of the right ventricle (arrow) into the right ventricle (arrowhead; asterisk indicates left ventricle). C, Microscopic section of a representative myocardial lesion. Rhabdomyocytes had loss of cross striations and were swollen and hyalinized (arrows). These changes were characteristic of acute coagulative necrosis (hematoxylin-eosin stain). D, Microscopic step section of the same myocardial lesion shown in C. Application of a histochemical stain for acute rhabdomyocte injury labeled the injured cells. The injured cells are stained dark (HBFP stain).

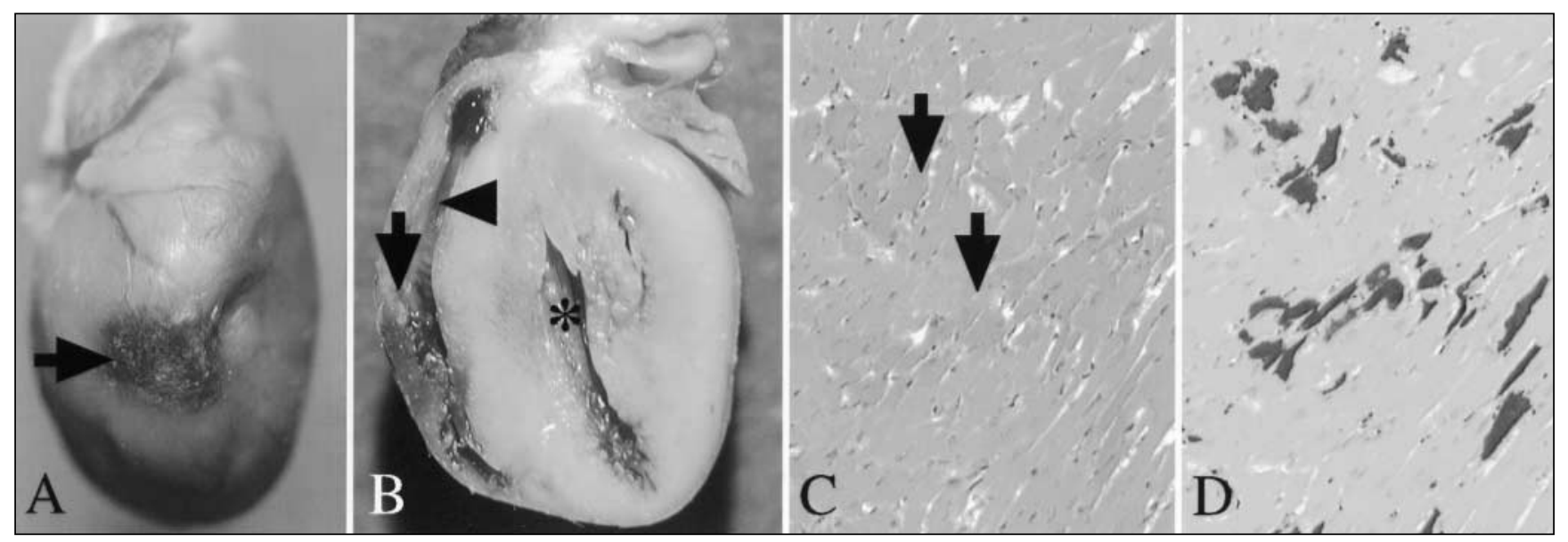


The premature contractions in frog hearts were hypothesized to be related to radiation force effects that were exerted directly on cells in the myocardium by the acoustic wave. ${ }^{38}$ Because Schelling et $\mathrm{al}^{41}$ have shown that extracorporeal shock waves can stimulate the initiation and conduction of electrical impulses in frog sciatic nerves, it is plausible that a mechanism that alters electrical activity within exposed hearts could be involved in the ventricular arrhythmias induced in our rats.

Of the known biomechanical phenomena associated with the interaction of the contrast agent with an ultrasonic field, inertial cavitation is the most plausible mechanism that could lead to development of arrhythmias. Bubble collapse might result in the formation of microjets of superheated gas, oxygen free radicals, peroxides, and other molecules toxic to cell membranes and cell organelles. ${ }^{42,43}$ Toxic molecules and microjets have the potential to cause direct injury to cells and cell membranes. Cellular aberrations in the transport or sequestration of intracellular and extracellular ions, such as calcium and potassium across membrane barriers and membrane systems, leading to changes in intracellular current potentials, must be considered as potential causes of ectopic foci. ${ }^{44}$

The exposure conditions used in this experiment were designed to test the hypothesis of whether arrhythmias could be induced in rat hearts exposed to ultrasound when a contrast agent was present in the circulatory system. Both the dose of the contrast agent administered intravenously $(0.25 \mathrm{~mL})$ and the MI (5.8) exceeded those used under normal conditions to obtain an echocardiogram in humans. Clinical protocols $^{1,4}$ for imaging left ventricle opacification and endocardial border delineation routinely use contrast agent volumes ranging between 0.2 and $5.0 \mathrm{~mL}$ and MIs between 0.2 and 0.8 , less then 1.9 as regulated by the FDA..$^{33}$ No clinically meaningful changes were observed in the electrocardiograms of 20 and 203 patients participating in these clinical studies. ${ }^{1,4}$ The volume of contrast agent based on body weight used in our study exceeded the volumes used in these clinical protocols by 14 to 345 times on the basis of body weight. The MI used in our study exceeded the indices used in those clinical protocols by 3 to 29 times. Even under these excessive experimental conditions, we were only able to produce meaningful arrhythmias in 10 of 20 rats. This result suggests that even at high exposure conditions, there are differences in susceptibility to the induction of arrhythmias. The factors that control these differences are unknown but could, in part, be due to transducer alignment issues as well as other biological and physiologic factors.

Studies need to be conducted to establish threshold levels for the initiation of arrhythmias by examining the roles of acoustic pressure (MI), contrast agent dose, contrast agent class, and exposure frequency. The mechanisms surrounding occurrence of ectopic electrical foci should be explored by examination of the role of alterations in ion balances and ion transport channels as well as the effects of drugs that inhibit arrhythmias and block ion transport pathways. These data will allow for a more clear understanding of the safety issues surrounding the use of contrast agents in at-risk patients.

\section{References}

1. Al-Mansour HA, Mulvagh SL, Pumper GM, Klarich $\mathrm{KW}$, Foley DA. Usefulness of harmonic imaging for left ventricular opacification and endocardial border delineation by Optison. Am J Cardiol 2000; 85:795799, A10.

2. Mulvagh SL, DeMaria AN, Feinstein SB, et al. Contrast echocardiography: current and future applications. J Am Soc Echocardiogr 2000; 13:331-342.

3. Clark LN, Dittrich HC. Cardiac imaging using Optison. Am J Cardiol 2000; 86:14G-18G.

4. Cohen JL, Cheirif J, Segar DS, et al. Improved left ventricular endocardial border delineation and opacification with OPTISON (FS069), a new echocardiographic contrast agent: results of a phase III multicenter trial. J Am Coll Cardiol 1998; 32:746-752.

5. Malhotra V, Nwogu J, Bondmass MD, et al. Is the technically limited echocardiographic study an endangered species? Endocardial border definition with native tissue harmonic imaging and Optison contrast: a review of 200 cases. J Am Soc Echocardiogr 2000; 13:771-773.

6. Miller JJ, Tiemann K, Podell S, et al. In vitro, animal, and human characterization of OPTISON infusions for myocardial contrast echocardiography. J Am Soc Echocardiogr 1999; 12:1027-1034.

7. Chomas JE, Dayton P, Allen J, Morgan K, Ferrara KW. Mechanisms of contrast agent destruction. IEEE Trans Ultrason Ferroelectr Freq Control 2001; 48:232-248. 
8. Brayman AA, Miller MW. Sonolysis of Albunexsupplemented, 40\% hematocrit human erythrocytes by pulsed 1-MHz ultrasound: pulse number, pulse duration and exposure vessel rotation dependence. Ultrasound Med Biol 1999; 25:307-314.

9. Dalecki D, Raeman CH, Child SZ, et al. Hemolysis in vivo from exposure to pulsed ultrasound. Ultrasound Med Biol 1997; 23:307-313.

10. Miller DL, Gies RA, Chrisler WB. Ultrasonically induced hemolysis at high cell and gas body concentrations in a thin-disc exposure chamber. Ultrasound Med Biol 1997; 23:625-633.

11. Miller DL, Gies RA. Enhancement of ultrasonicallyinduced hemolysis by perfluorocarbon-based compared to air-based echo-contrast agents. Ultrasound Med Biol 1998; 24:285-292.

12. Poliachik SL, Chandler WL, Mourad PD, et al. Effect of high-intensity focused ultrasound on whole blood with and without microbubble contrast agent. Ultrasound Med Biol 1999; 25:991-998.

13. Williams AR, Kubowicz G, Cramer E, Schlief R. The effects of the microbubble suspension SH U 454 (Echovist) on ultrasound-induced cell lysis in a rotating tube exposure system. Echocardiography 1991; 8:423-433

14. Brayman AA, Lizotte LM, Miller MW. Erosion of artificial endothelia in vitro by pulsed ultrasound: acoustic pressure, frequency, membrane orientation and microbubble contrast agent dependence. Ultrasound Med Biol 1999; 25:1305-1320.

15. Miller DL, Bao S. The relationship of scattered subharmonic, 3.3-MHz fundamental and second harmonic signals to damage of monolayer cells by ultrasonically activated Albunex. J Acoust Soc Am 1998; 103:1183-1189.

16. Miller DL, Quddus J. Sonoporation of monolayer cells by diagnostic ultrasound activation of contrastagent gas bodies. Ultrasound Med Biol 2000; 26: 661-667.

17. Ward M, Wu J, Chiu JF. Ultrasound-induced cell lysis and sonoporation enhanced by contrast agents. J Acoust Soc Am 1999; 105:2951-2957.

18. Miller DL, Quddus J. Diagnostic ultrasound activation of contrast agent gas bodies induces capillary rupture in mice. Proc Natl Acad Sci USA 2000; 97: 10179-10184.
19. Miller DL, Gies RA. The influence of ultrasound frequency and gas-body composition on the contrast agent-mediated enhancement of vascular bioeffects in mouse intestine. Ultrasound Med Biol 2000; 26:307-313.

20. Skyba DM, Price RJ, Linka AZ, Skalak TC, Kaul, S. Direct in vivo visualization of intravascular destruction of microbubbles by ultrasound and its local effects on tissue. Circulation 1998; 98:290-293.

21. American Institute of Ultrasound in Medicine. Clinical relevance. In: Mechanical Bioeffects From Diagnostic Ultrasound: AIUM Consensus Statements. J Ultrasound Med 2000; 19:149-153.

22. American Institute of Ultrasound in Medicine. Conclusions and recommendations. In: Mechanical Bioeffects From Diagnostic Ultrasound: AIUM Consensus Statements. J Ultrasound Med 2000; 19:73-76.

23. van Der Wouw PA, Brauns AC, Bailey SE, Powers JE, Wilde AA. Premature ventricular contractions during triggered imaging with ultrasound contrast. J Am Soc Echocardiogr 2000; 13:288-294.

24. O'Brien WD Jr, Simpson DG, Frizzell LA, Zachary JF. Superthreshold behavior and threshold estimation of ultrasound-induced lung hemorrhage in adult rats: role of beam width. IEEE Trans Ultrason Ferroelectr Freq Control 2001; 48:1695-1705.

25. O'Brien WD Jr, Schaeffer DJ, Frizzell LA, Zachary JF. Superthreshold behavior of ultrasound-induced lung hemorrhage in adult mice and rats: role of pulse repetition frequency and pulse duration. Ultrasound Med Biol 2001; 27:267-277.

26. Zachary JF, Frizzell LA, Norrell KS, Blue JP, Miller RJ, O'Brien WD Jr. Temporal and spatial evaluation of lesion resolution following exposure of rat lung to pulsed ultrasound. Ultrasound Med Biol 2001; 27:829-839.

27. Zachary JF, Semsprott JM, Frizzell LA, Simpson DG, O'Brien WD Jr. Superthreshold behavior and threshold estimation of ultrasound-induced lung hemorrhage in mice and rats. IEEE Trans Ultrason Ferroelectr Freq Control 2001; 48:581-592.

28. Podell S, Burrascano C, Gaal M, Golec B, Maniquis J, Mehlhaff P. Physical and biochemical stability of Optison, an injectable ultrasound contrast agent. Biotechnol Appl Biochem 1999; 30:213-223. 
29. Optison (human albumin microsphere injectable suspension octafluoropropane formulation) [package insert]. St Louis, MO: Mallinckrodt Inc; 1998.

30. Lie JT, Holley KE, Kampa WR, Titus JL. New histochemical method for morphologic diagnosis of early stages of myocardial ischemia. Mayo Clin Proc 1971; 46:319-327.

31. Lie JT, Holley KE, Titus JL. Fuchsinorrhagia: a new histochemical indication of inapparent early myocardial ischemia. Lab Med 1972; 37:37-40.

32. Lie JT, Titus JL. Pathology of the myocardium and the conduction system in sudden coronary death. Circulation 1975; 52:41-52.

33. Raum K, O'Brien WD Jr. Pulse-echo field distribution measurement technique of high-frequency ultrasound sources. IEEE Trans Ultrason Ferroelectr Freq Control 1997; 44:810-815.

34. Teotico GA, Miller RJ, Frizzell LA, Zachary JF, O'Brien WD Jr. Attenuation coefficient estimates of mouse and rat chest wall. IEEE Trans Ultrason Ferroelectr Freq Control 2001; 48:583-601.

35. Dalecki D, Keller BB, Raeman CH, Carstensen EL. Effects of pulsed ultrasound on the frog heart, I: thresholds for changes in cardiac rhythm and aortic pressure. Ultrasound Med Biol 1993; 19:385-390.

36. Food and Drug Administration. Information for Manufacturers Seeking Marketing Clearance of Diagnostic Ultrasound Systems and Transducers. Rockville, MD: Center for Devices and Radiological Health, US Food and Drug Administration; 1997.

37. Dalecki D, Raeman CH, Carstensen EL. Effects of pulsed ultrasound on the frog heart, II: an investigation of heating as a potential mechanism. Ultrasound Med Biol 1993; 19:391-398.

38. Dalecki D, Raeman CH, Child SZ, Carstensen EL. Effects of pulsed ultrasound on the frog heart, III: the radiation force mechanism. Ultrasound Med Biol 1997; 23:275-285.

39. MacRobbie AG, Raeman CH, Child SZ, Dalecki D. Thresholds for premature contractions in murine hearts exposed to pulsed ultrasound. Ultrasound Med Biol 1997; 23:761-765.

40. Delius M, Hoffmann E, Steinbeck G, Conzen P. Biological effects of shock waves: induction of arrhythmia in piglet hearts. Ultrasound Med Biol 1994; 20:279-285.
41. Schelling G, Delius M, Gschwender M, Grafe P, Gambihler S. Extracorporeal shock waves stimulate frog sciatic nerves indirectly via a cavitation-mediated mechanism. Biophys J 1994; 66:133-140.

42. American Institute of Ultrasound in Medicine. Definitions and descriptions of nonthermal mechanisms. In: Mechanical Bioeffects From Diagnostic Ultrasound: AIUM Consensus Statements. J Ultrasound Med 2000; 19:77-84.

43. American Institute of Ultrasound in Medicine. Mechanical bioeffects in the presence of gas-carrier ultrasound contrast agents. In: Mechanical Bioeffects From Diagnostic Ultrasound: AIUM Consensus Statements. J Ultrasound Med 2000; 19:120-142.

44. Fareh S, Benardeau A, Nattel S. Differential efficacy of L- and T-type calcium channel blockers in preventing tachycardia-induced atrial remodeling in dogs. Cardiovasc Res 2001; 49:762-770. 